\title{
AN INVARIANCE PRINCIPLE FOR THE EMPIRICAL PROCESS WITH RANDOM SAMPLE SIZE
}

\author{
BY M. CSÖRGÖ ${ }^{1}$ AND S. CSÖRGÖ
}

Communicated by David Blackwell, January 19, 1970

Let $C=C[0,1]$ be the space of continuous functions on $[0,1]$ with the uniform topology, that is the distance between two points $x$ and $y$ (two functions $x$ and $y$ of $t \in[0,1]$ ) is defined by

$$
\rho(x, y)=\sup _{t}|x(t)-y(t)| .
$$

Let $B$ be the $\sigma$-field of Borel sets of $C$.

Let $(\Omega, Q, P)$ be some probability space and $W$ be the Wiener measure on $(C, B)$ with the corresponding Wiener process $\left\{W_{t}(\omega): 0 \leqq t \leqq 1\right\}, \omega \in \Omega$; that is $W_{t}$ has values in $C$ and is specified by $E\left(W_{t}\right)=0$ and $E\left(W_{s} W_{t}\right)=s$ if $s \leqq t$. Let $W^{0}$ be the Gaussian measure on $(C, B)$ constructed by setting $W_{t}^{0}=W_{t}-t W_{1}$. Then $W_{t}^{0} \in C$, $E\left(W_{t}^{0}\right)=0$ and $E\left(W_{s}^{0} W_{l}^{0}\right)=s(1-t)$ if $s \leqq t$. Also $W_{0}^{0}=W_{1}^{0}=0$ with probability 1 and $\left\{W_{t}^{0}: 0 \leqq t \leqq 1\right\}$ is called the tied down Wiener process or the Brownian bridge.

Let $S_{n}=\xi_{1}+\cdots+\xi_{n}, S_{0}=0, n=1,2, \cdots$ be the partial sum sequence of random variables $\left\{\xi_{n}\right\}$ defined on $(\Omega, a, P)$. Define a random element $X_{n}$ of $C$ by

(1) $X_{n}(t, \omega)=W_{n}(t, \omega)+(n t-[n t]) \xi_{[n t]+1}(\omega) / n^{1 / 2}-t W_{n}(1, \omega)$

where $W_{n}(t, \omega)=S_{[n t]}(\omega) / n^{1 / 2}$. The following theorem is an immediate consequence of L. Breiman's analysis of $\$ \$ 13.5$ and 13.6 in his book [3].

Theorem B. Suppose the random variables $\xi_{1}, \xi_{2}, \ldots$ are independent and identically distributed with mean zero and variance 1 . Then the random functions $X_{n}$ defined by (1) satisfy

$$
X_{n} \stackrel{D}{\rightarrow} W^{0} .
$$

Here (2), and also similar relations later on, are interpreted in accordance with (4.5) and (4.7) of Billingsley's book [2], depending on

AMS Subject Classifications. Primary 6030, 6040; Secondary 6270, 6271.

Key Words and Phrases. Invariance principle, weak convergence, randomly selected partial sums, empirical process, random-sample-size Kolmogorov-Smirnov statistics.

${ }^{1}$ Work done while the author was a Canada Council Fellow in 1969-1970 at the University of Vienna. 
whether $W^{0}$ is construed as a random function or as a measure in the spirit of $[2$, p. 65]; the meaning is the same for the two interpretations. Since $h(x)=\sup _{t}|x(t)|$ with $x(t)=w(t)-t w(1)$ is a continuous function on $C$ in the sup-norm metric, (2) implies

$$
\sup _{t}\left|X_{n}(t)\right| \stackrel{D}{\rightarrow} \sup _{t}\left|W_{t}^{0}\right|
$$

an invariance principle, as statements like this are often called. Similarly,

$$
\sup _{t} X_{n}(t) \stackrel{\mathbb{D}}{\rightarrow} \sup _{t} W_{t}^{0}, \quad \inf X_{t}(t) \stackrel{\mathbb{D}}{\rightarrow} \inf _{t} W_{t}^{0} .
$$

For each $n$, let $\nu_{n}$ be a positive-integer-valued random variable defined on the same probability space as the $\xi_{n}$. Define $X_{n}$, a random element of $C$, as in (1), and $Y_{n}$, another random element of $C$, by

$$
Y_{n}(t, \omega)=X_{\nu_{n}(\omega)}(t, \omega) .
$$

TheOREM 1. Suppose the random variables $\xi_{1}, \xi_{2}, \ldots$ are independent and identically distributed with mean zero and variance 1 . If

$$
\nu_{n} / n \stackrel{P}{\rightarrow} \nu,
$$

where $\nu$ is a positive random variable, and

$$
\xi_{\left[\nu_{n} t\right]}(\omega) /\left(\nu_{n}(\omega)\right)^{1 / 2} \stackrel{P}{\rightarrow} 0, \quad \text { for every fixed } t,
$$

then the random functions $Y_{n}$ defined by (3) satisfy

$$
Y_{n} \stackrel{D}{\rightarrow} W^{0} .
$$

CoROllary 1. Under the same assumptions as in Theorem 1 (6) implies

$$
\begin{gathered}
\sup _{t}\left|Y_{n}(t)\right| \stackrel{\mathcal{D}}{\rightarrow} \sup _{t}\left|W_{t}^{0}\right|, \\
\sup _{t} Y_{n}(t) \stackrel{D}{\rightarrow} \sup _{t} W_{t}^{0}, \\
\quad \inf _{t} Y_{n}(t) \stackrel{D}{\rightarrow} \inf _{t} W_{t}^{0} .
\end{gathered}
$$

Remark 1. Let $D$ be the space $D$ of Chapter 3 of P. Billingsley's book [2]. Define random elements $X_{n}^{*}, Y_{n}^{*}$ of $D$ by 


$$
\begin{aligned}
X_{n}^{*}(t, \omega) & =W_{n}(t, \omega)-t W_{n}(1, \omega), \\
Y_{n}^{*}(t, \omega) & =X_{\nu_{n}(\omega)}^{*}(t, \omega)
\end{aligned}
$$

with $W_{n}(t, \omega)$ as in (1). Then Theorem B holds for $X_{n}^{*}$ of $(7)$ and, omitting condition (5), Theorem 1 holds for $Y_{n}^{*}$ of (8). Also, in defining $Y_{n}$ of (3) and $Y_{n}^{*}$ of (8) it is not essential that the random variables $\left\{\xi_{n}\right\}$ involved should be independent and identically distributed with unit variance. We have stated Theorem 1 here for random elements of $C$ and for independent identically distributed random variables having unit variance only because it is, as will be shown later, directly applicable in this form to prove the random-sample-size Kolmogorov-Smirnov theorems. More general versions of Theorem 1 and detailed proofs of them will appear in [4]. We also note that for $Y_{n}$ of (3) one postulates (5), for it is not true in general that $\xi_{[n t]} / n^{1 / 2}$ $\stackrel{P}{\rightarrow} 0$ and $(4)$ imply (5).

For the proof of Theorem 1 we use Theorem B, Theorems 7.7, 8.1, 8.2 of P. Billingsley's book [2] and results of A. Rényi [7] and J. Mogyoródi [5]. First we show that for a single time point $s\left\{X_{n}^{*}(s)\right\}$ is mixing with the normal distribution function $N(0, s(1-s))$ in the sense of A. Rényi's definition of mixing sequences of events [7] and that it also satisfies the tightness condition of F. J. Anscombe [1]. Then, using Theorem B, Theorem 7.7 of [2] and Theorem 2 of [5], we show that the finite-dimensional distributions of $Y_{n}$ of (3) converge to those of $W^{0}$. Next it is verified that the sequence $\left\{Y_{n}\right\}$ is tight in the sense of Theorem 8.2 of [2] and then Theorem 1 follows from Theorem 8.1 of [2]. Details of this proof will appear in [4].

Let $U_{1}, \cdots, U_{n}$ be independent random variables uniformly distributed on $[0,1]$. The order statistics are defined as follows: $U_{1}^{(n)}$ is the smallest, and so forth; $U_{n}^{(n)}$ is the largest. Let

$$
F_{n}(t)=\left(\text { the number of the } U_{i} \leqq t\right) / n, \quad t \in[0,1] .
$$

Define the Kolmogorov-Smirnov statistics

$$
\begin{aligned}
& D_{n}^{+}=n^{1 / 2} \sup _{t}\left(F_{n}(t)-t\right)=n^{1 / 2} \max _{k \leqq n}\left(k / n-U_{k}^{(n)}\right), \\
& D_{n}^{-}=n^{1 / 2} \inf _{t}\left(F_{n}(t)-t\right)=n^{1 / 2} \min _{k \leqq n}\left(k / n-U_{k}^{(n)}\right), \\
& D_{n}=n^{1 / 2} \sup _{t}\left|t-F_{n}(t)\right|=n^{1 / 2} \max _{k \leqq n}\left|U_{k}^{(n)}-k / n\right|,
\end{aligned}
$$

and the random-sample-size Kolmogorov-Smirnov statistics $\Delta_{n}^{+}$ $=D_{\nu_{n}}^{+}, \Delta_{n}^{-}=D_{v_{n}}^{-}, \Delta_{n}=D_{v_{n}}$. 
THEOREM 2. Under condition (4) of Theorem 1 we have

$$
\Delta_{n}^{+} \stackrel{D}{\rightarrow} \sup _{t} W_{t}^{0}, \quad \Delta_{n}^{-} \stackrel{D}{\rightarrow} \inf _{t} W_{t}^{0}, \quad \Delta_{n} \stackrel{D}{\rightarrow} \sup _{t}\left|W_{t}^{0}\right| .
$$

Proof of Theorem 2. Let $S(n)=\zeta_{1}+\cdots+\zeta_{n}, n=1,2, \cdots$ be the partial sum sequence of independent exponential random variables $\left\{\zeta_{n}\right\}$ with mean 1. L. Breiman $[3, \S 13.6]$ obtained the following representation of $D_{n}$

$$
\begin{aligned}
D_{n} & \stackrel{D}{=} n^{1 / 2} \max _{k \geqq n}\left|\frac{S(k)}{S(n+1)}-\frac{k}{n}\right| \\
& \stackrel{D}{=} \frac{n}{S(n+1)} \max _{k \leqq n}\left|\frac{S(k)-k}{n^{1 / 2}}-\frac{k}{n} \frac{S(n+1)-n}{n^{1 / 2}}\right|,
\end{aligned}
$$

with analogous expressions for $D_{n}^{+}$and $D_{n}^{-}$. Here $\stackrel{D}{=}$ means that the random variables in question have the same distribution. Put $\xi_{n}=\zeta_{n}-1, S_{k}=S(k)-k$ and $W_{n}(t, \omega)=S_{[n t]}(\omega) / n^{1 / 2}$. Then

$$
\begin{aligned}
D_{n} & \stackrel{D}{=} \sup _{t}\left|X_{n}^{*}(t, \omega)\right|, \quad \text { for } n \text { large, } \\
& \stackrel{D}{=} \sup _{t}\left|X_{n}(t, \omega)\right|, \quad \text { for } n \text { large, }
\end{aligned}
$$

where $X_{n}^{*}$ and $X_{n}$ are respectively defined in terms of the above $\xi_{n}$ and $W_{n}$ via (7) and (1). Analogous asymptotic representations hold for $D_{n}^{+}$and $D_{n}^{-}$. The first asymptotic representation of (10) for $D_{n}$ is true because $E\left(\zeta_{n}\right)=\sigma^{2}\left(\zeta_{n}\right)=1$ and hence $n / S(n+1) \stackrel{\text { a.s. }}{\rightarrow} 1$ and $\zeta_{n+1} / n^{1 / 2} \stackrel{P}{\rightarrow} 0$, while the second asymptotic representation of (10) is the consequence of $\xi_{[n t]+1} / n^{1 / 2} \stackrel{P}{\rightarrow} 0$ uniformly in $t$. The $X_{n}$ of (10) satisfy the conditions of Theorem B and the usual Kolmogorov-Smirnov theorems follow. For $\Delta_{n}$ we have (9) with $n$ replaced by $\nu_{n}$ on both sides. Now we show

$$
\begin{aligned}
& \Delta_{n} \stackrel{D}{=} \sup _{t}\left|Y_{n}^{*}(t, \omega)\right|, \quad \text { for } n \text { large, } \\
& \stackrel{D}{=} \sup _{t}\left|Y_{n}(t, \omega)\right|, \quad \text { for } n \text { large, }
\end{aligned}
$$

where $Y_{n}^{*}$ and $Y_{n}$ are respectively defined in terms of the above $\xi_{n}$ and $W_{n}$ via (8) and (3); we also have the analogous asymptotic expressions for $\Delta_{n}^{+}$and $\Delta_{n}^{-}$. It is true in general that if $\left\{Z_{n}\right\}$ is a sequence of random variables such that $Z_{n} \stackrel{\text { a.s. }}{\longrightarrow} Z$ and $\left\{\nu_{n}\right\}$ is a sequence of 
positive-integer-valued random variables such that $\nu_{n} \stackrel{P}{\rightarrow}+\infty$, then $Z_{\nu_{n}} \stackrel{P}{\rightarrow} Z$. Now condition (4) of Theorem 1 implies $\nu_{n} \stackrel{P}{\rightarrow}+\infty$ and we have $n / S(n+1) \stackrel{\text { a.s. }}{\rightarrow} 1$. Consequently, $\nu_{n} / S\left(\nu_{n}+1\right) \stackrel{P}{\rightarrow} 1$. Using the fact that the $\zeta_{n}$ are exponential random variables with mean 1 and that $\nu_{n} \stackrel{P}{\rightarrow}+\infty$, it can be easily shown that $\zeta_{\nu_{n}+1} / \nu_{n}^{1 / 2}$ and $\xi_{\left[\nu_{n} t\right]+1} / \nu_{n}^{1 / 2}$ both converge in probability to zero, the latter one uniformly in $t$. Hence both asymptotic representations of (11) are true. Also, given condition (4), the $Y_{n}$ of (11) satisfy the conditions of Theorem 1 and hence $Y_{n} \stackrel{D}{\rightarrow} W^{0}$. The statements of Theorem 2 now follow from Corollary 1 .

REMARK 2. Theorem 2 with $\nu=1$ in (4) was proved by R. Pyke [6] in an interesting and different way, utilizing results about stochastic processes with two-dimensional parameter sets. We should also note that proving appropriate versions of Theorem 1, random-sample-size versions of the Kolmogorov-Smirnov theorems with weight functions like

$$
f(t)=1 / t, \quad 1 /(1-t) \text { and } 1 /[t(1-t)]^{1 / 2} \text {, }
$$

which are important in applications, can also be proved in a similar way as well as two or more-sample random-sample-size versions. Statements and proofs for these results will also appear in [4].

\section{REFERENCES}

1. F. J. Anscombe, Large-sample theory of sequential estimation, Proc. Cambridge Philos. Soc. 48 (1952), 600-607. MR 14, 487.

2. P. Billingsley, Convergence of probability measures, Wiley, New York, 1968. MR $38 \# 1718$.

3. L. Breiman, Probability, Addison-Wesley, Reading, Mass., 1968. MR 37 \#4841.

4. M. Csörgö and S. Csörgö, On weak convergence of randomly selected partial sums (to appear).

5. J. Mogyorodi, Limit distributions for sequences of random variables with random indices, Trans. Fourth Prague Conf. on Information Theory, Statistical Decision Functions, Random Processes, (Prague, 1965) Academia, Prague, 1967, pp. 463-470. MR 36 \#935.

6. R. Pyke, The weak convergence of the empirical process with random sample size, Proc. Cambridge Philos. Soc. 64 (1968), 155-160. MR 36 \#3402.

7. A. Rényi, On mixing sequences of sets, Acta. Math. Acad. Sci. Hungar. 9 (1958), 215-228. MR 20 \#4623.

McGill University, Montreal, Quebec, Canada

József Attila University, Szeged, Hungary

Mathematisches Institut der Universitat, Wien, Austria 